\title{
\begin{tabular}{l|l} 
Mibraries & DSpace@MIT
\end{tabular}
}

\author{
MIT Open Access Articles
}

Astro-H data analysis, processing and archive

The MIT Faculty has made this article openly available. Please share how this access benefits you. Your story matters.

Citation: Angelini, Lorella, et al. "Astro-H Data Analysis, Processing and Archive." Proceedings Volume 9905, Space Telescopes and Instrumentation 2016: Ultraviolet to Gamma Ray, 29 June - 1 July, 2016, Edinburgh, Scotland, edited by Jan-Willem A. den Herder et al., SPIE, 2016, p. 990514. (c) 2016 SPIE

As Published: http://dx.doi.org/10.1117/12.2234429

Publisher: SPIE

Persistent URL: http://hdl.handle.net/1721.1/114869

Version: Final published version: final published article, as it appeared in a journal, conference proceedings, or other formally published context

Terms of Use: Article is made available in accordance with the publisher's policy and may be subject to US copyright law. Please refer to the publisher's site for terms of use. 


\section{Astro-H data analysis, processing and archive}

Lorella Angelini, Yukitatsu Terada, Michael Loewenstein, Eric D. Miller, Hiroya Yamaguchi, et al.

Lorella Angelini, Yukitatsu Terada, Michael Loewenstein, Eric D. Miller, Hiroya Yamaguchi, Tahir Yaqoob, Hans Krimm, Ilana Harrus, Hiromitsu Takahashi, Masayoshi Nobukawa, Makoto Sawada, Michael Witthoeft, Kristin Rutkowski, Andrew Sargent, Robert S. Hill, Michael Dutka, Joseph Eggen, "Astro-H data analysis, processing and archive," Proc. SPIE 9905, Space Telescopes and Instrumentation 2016: Ultraviolet to Gamma Ray, 990514 (15 July 2016); doi: 10.1117/12.2234429

Event: SPIE Astronomical Telescopes + Instrumentation, 2016, Edinburgh, United Kingdom 


\title{
Astro-H data analysis, processing and archive
}

Lorella Angelini*a $^{*}$, Yukikatsu Terada ${ }^{b}$, Michael Loewenstein ${ }^{\text {a,c }}$, Eric D. Miller ${ }^{\mathrm{d}}$, Hiroya Yamaguchi $^{\text {a,c }}$, Tahir Yaqoob ${ }^{\text {a,e }}$, Hans Krimm ${ }^{\text {a,f }}$, Ilana Harrus ${ }^{\text {a,e }}$,

Hiromitsu Takahashi ${ }^{\mathrm{g}}$, Masayoshi Nobukawa ${ }^{\mathrm{h}}$, Makoto Sawada ${ }^{\mathrm{i}}$, Michael Witthoeft ${ }^{\mathrm{j}}$,

Kristin Rutkowski ${ }^{j}$, Andrew Sargent ${ }^{k}$, Robert S. Hill ${ }^{j}$, Michael Dutka ${ }^{k}$, Joseph Eggen ${ }^{j}$

${ }^{a}$ Goddard Space Flight Center NASA, 8800 Greenbelt Road, Greenbelt MD 20771; ${ }^{\mathrm{b}}$ Saitama

University, 255 Shimookubo, Sakura, Saitama, Saitama Prefecture, 338-8570, Japan; ${ }^{\circ}$ Department of

Astronomy, University of Maryland, College Park, MD 20742, USA; ${ }^{\mathrm{d} M I T}$ Kavli Institute for

Astrophysics and Space Research Laboratory 77 Massachusetts Ave, Cambridge, MA 02139;

${ }^{e}$ Department of Physics, University of Maryland Baltimore County, Baltimore, MD 21250, USA;

${ }^{f}$ Universities Space Research Association, 10211 Wincopin Circle, Suite 500, Columbia, MD 21044, USA; ${ }^{\mathrm{g}}$ Hiroshima University, 1-3-1, Kagamiyama, Higashi-Hiroshima, Hiroshima Prefecture, 739-

8526, Japan ; ${ }^{\mathrm{h}}$ Nara University of Education, Takabatake-cho, Nara, Nara Prefecture, 630-8528, Japan; ${ }^{\mathrm{I} A o y a m a}$ Gakuin University, 5-10-1 Fuchinobe, Chuo-ku, Sagamihara,Kanagawa Prefecture, 252-5258, Japan; ${ }^{j}$ ADNET Systems 6720 Rockledge Drive Suite 504 Bethesda, MD 20817, USA;

${ }^{k}$ Wyle 7315 Mission Drive Lanham, MD 20706, USA.

\begin{abstract}
Astro-H (Hitomi) is an X-ray/Gamma-ray mission led by Japan with international participation, launched on February 17, 2016. The payload consists of four different instruments (SXS, SXI, HXI and SGD) that operate simultaneously to cover the energy range from $0.3 \mathrm{keV}$ up to $600 \mathrm{keV}$. This paper presents the analysis software and the data processing pipeline created to calibrate and analyze the Hitomi science data along with the plan for the archive and user support. These activities have been a collaborative effort shared between scientists and software engineers working in several institutes in Japan and USA.
\end{abstract}

Keywords: Astro-H, Software, Calibration, Pipeline, Archive

\section{INTRODUCTION}

Astro- $\mathrm{H}^{1}$, renamed Hitomi after launch, is a facility-class mission launched on a JAXA H-IIA rocket into low Earth orbit on Feb 17, 2016 at 5:45 pm JPS from Tanegashima Space Center in Japan. Hitomi is Japan's sixth X-ray astronomy mission primarily developed at the Institute of Space and Astronautical Science of Japan Aerospace Exploration Agency (ISAS/JAXA) in collaboration with U.S. (NASA/GSFC) and Japanese institutions, with contributions from the European Space Agency (ESA), the Netherlands Institute for Space Research (SRON), the Canadian Space Agency (CSA) and US and European institutions. Hitomi is equipped with four different instruments that together cover a wide energy range $0.3-600 \mathrm{keV}$. The Soft X-ray Spectrometer $\left(\mathrm{SXS}^{2}\right.$ ), which pairs a lightweight Soft X-ray Telescope ${ }^{3}$ with a X-ray Calorimeter Spectrometer, provides non-dispersive spectroscopy with $<7 \mathrm{eV}$ resolution in the $0.3-10 \mathrm{keV}$ energy range with a field of view of about 3 arcmin. Three additional scientific instruments extend the energy bandpass of the observatory. The Soft X-ray Imager (SXI ${ }^{4}$ ) expands the field of view with a new generation CCD camera in the energy range of $0.5-12 \mathrm{keV}$ at the focus of the second lightweight Soft X-ray Telescope ${ }^{3}$; the Hard X-ray Imager (HXI ${ }^{5.6}$, two units) performs sensitive imaging spectroscopy in the $5-80 \mathrm{keV}$ band; the nonimaging Soft Gamma-ray Detector (SGD ${ }^{7}$, two units) extends Hitomi's energy band to $600 \mathrm{keV}$. On March 27, 2016, JAXA lost contact with the satellite, and on April 28 announced that they would cease the efforts to restore the mission operations. The Hitomi instruments collected about one month worth of data that are processed and archived.

The following sections describe the Hitomi data format, the software developed to calibrate and analyze the science data, how the data are processed on ground, the different levels of data created and how they are archived. The latest updates on software, calibration and archives are posted at http://heasarc.gsfc.nasa.gov/docs/hitomi/ .

*lorella.angelini-1@nasa.gov; $\quad$ phone 1301 286-3607; fax $1301286-0677$

Space Telescopes and Instrumentation 2016: Ultraviolet to Gamma Ray, edited by Jan-Willem A. den Herder, Tadayuki Takahashi, Marshall Bautz, Proc. of SPIE Vol. 9905, 990514

(C) 2016 SPIE · CCC code: 0277-786X/16/\$18 - doi: 10.1117/12.2234429 


\section{DESIGN OF THE FITS FILES, SOFTWARE, PROCESSING AND ARCHIVE}

The design of the ground processing has been developed with the same guiding principles and models used by previous high energy astrophysics missions such as ASCA, RXTE, Swift and Suzaku. The telemetry data are reformatted in FITS format at the early stage and the analysis software is working directly on these FITS files. The FITS files are designed following the HEASARC/OGIP FITS conventions. The Hitomi software is built within the HEAsoft environment and distributed by the HEASARC. HEAsoft does not depend on any specific environment and it is supported on many operating systems commonly used in high energy astrophysics. The calibration data are all ingested in the HEASARC Calibration Database (CALDB). The calibration information is stored in files, also in FITS format, and interface with the software via routines that query the CALDB metadata. The pipeline uses the same environment of the data pipeline for ASCA, SWIFT and Suzaku and the data processing uses the Hitomi software distributed with HEAsoft.

\subsection{Data division and FITS format}

The Hitomi data are organized by sequence number and placed in the archive after processing. A sequence number may contain the entire observation on an object if shorter than one day. Observations longer than a day are divided into different sequences on the boundary between days from the start time of the observation (Figure 1). Each observation contains both pointing and incoming slew data for all instruments, subsystems and housekeeping. The science files for each instrument are divided into slew and pointing data. The science data files may be further divided by the instrument mode or filter. The instrument and spacecraft housekeeping (HK), as well as the attitude and orbit, data are instead not separated by slew and pointing.

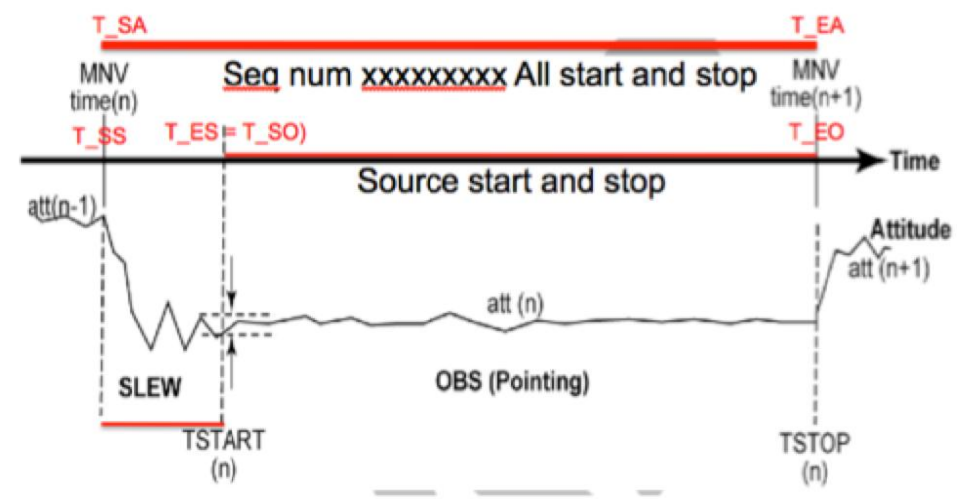

Figure 1. The data within a sequence are all that occurs between T_SA and T_EA, which indicate the start and end of the attitude. Within these times, the science data are divided in slew and pointing.

All Hitomi telemetry data within a sequence are reformatted in FITS without loss of information, using either the BINTABLE or IMAGE extensions. The science data are organized in FITS BINTABLE using the EVENT format where each row contains several columns with information specific to an event. The HK data are in time ordered FITS BINTABLE tables recording various parameters related to the instruments and subsystems. The files generated from the telemetry are named First FITS Files (FFF), and after calibration are named Second FITS File (SFF and SFFa). The science file for each of the instruments contains different types of signals related either to the calibration sources or electronic signals used to derive corrections. The SXS and SXI files contain columns with either a single element or fixed array in one row with information related to one event. The FFF files for the HXI and SGD instead contain variable length arrays. The X-ray interaction with the HXI or SGD detectors at any one time produces an ensemble of signals. The number of signals in one interaction (hereafter "occurrence") is variable and stored in variable length array columns. The path of these signals within the detectors, belonging to an occurrence, is reconstructed to an event. The reconstructed events for the HXI and SGD are stored in the SFFa files that do not have variable length array columns.

\subsection{Software and Calibration data}

The calibration and analysis of the Hitomi data requires mission-specific tasks, as well as multi-mission tasks included within HEASoft (http://heasarc.gsfc.nasa.gov/docs/software/lheasoft/ ). The Hitomi-specific tasks consist of 78 tools to 
support various stages of the calibration, data filtering, as well as simulation for all instrument and subsystems. Each task is built as an FTOOL (standard within HEAsoft) and uses a standard parameter file as interface, the CFITSIO library to access and write FITS files and a common makefile to build the code. Each of the Hitomi tasks is dedicated to a specific function and written in $\mathrm{C}$ or $\mathrm{C}++$ or perl.

The tasks for the instrument calibration include the assignment of time, energy and coordinates. The calibration of the subsystems are for the Canadian Astro-H Metrology System (CAMS ${ }^{8}$ ), used to calculate the coordinates for the HXI, and for the Modulated X-ray Source (MXS ${ }^{9}$ ), used for time dependent gain correction of the SXS data. There are tasks to calculate an auxiliary response file (ARF, the effective area of the telescope which also includes the detector properties), instrument response matrix file (RMF or RSP) and exposure map. Several scripts facilitate the data calibration and data filtering. Ray-tracing code has been developed to model the telescopes, and a general simulator to generate events for a specific source and to model the sky background. The analysis of the Hitomi data makes use of standard multi-mission software packages existing within HEASoft: Xselect allows to extract events, light curves and /or spectra within a region and /or specific pulse phases or source intensity; Xspec, Xronos and Ximage are for spectral, timing, and imaging analysis.

The Hitomi software uses the HEASARC CALDB (http://heasarc.gsfc.nasa.gov/docs/heasarc/caldb/caldb intro.html) to access the Hitomi calibration information. The Hitomi calibration data is in FITS files and includes all the pre-launch information as well as the post launch updates. The calibration files are designed to accommodate dependencies either on time or specific mode or other parameters to allow software to retrieve the correct file via the CALDB metadata that lists these dependences. The updates of the calibration information do not require changes in the software but only updates of the CALDB files and metadata.

\subsection{Data flow: pre-pipeline, pipeline and archive}

The processing data flow consists of two steps: the pre-pipeline (run at ISAS Japan) that decodes the telemetry data and the pipeline (run at GSFC/USA) that calibrates the data and transfers the data to the archives (Figure 2).

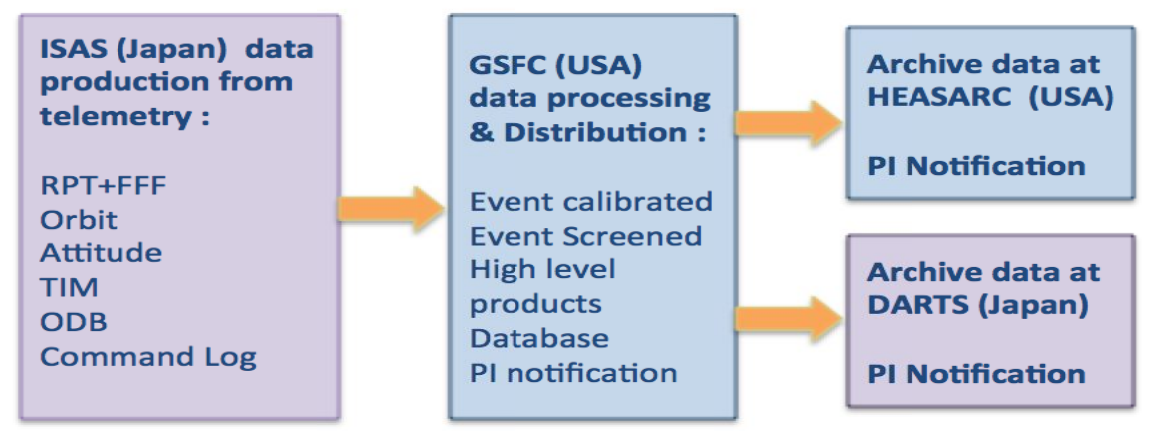

Figure 2. The data processing flow. Pre-pipeline for data preparation, pipeline for data processing and archive.

In the pre-pipeline the Hitomi telemetry are divided into observations, labeled with a sequence number, and stored in files with a FITS wrapper named Raw Package Telemetry (RPT). The FFF files are created by decoding the telemetry from the RPT together with other support files, such as those for the attitude and orbit (Figure 3). These files have the structure and the number of columns of the final FITS files, but the pre-pipeline only populates the columns with the telemetered values and as an exception the TIME column to ensure that the attitude and orbit data are also time tagged consistently. The pipeline populates the remaining columns in the FITS file with the calibrated information. The data processing in the pipeline creates: a) the unfiltered calibrated data (SFF file also Level 1/1a) where the information for all events is maintained; b) the good time intervals obtained excluding unwanted times either due to orbital or instrument HK parameters; c) the filtered or cleaned files (Level 2) where the data are screened for unwanted event flags and selected within the good time intervals; d) high level products such as images, spectra, and light curves for all instruments; e) additional files to monitor the instrument performance; and f) the database file containing high level information for each observation used by the archive. The tasks used to process the data in the pipeline as well as to calculate the timing information used in the pre-pipeline are all part of the Hitomi package so that users may always recalibrate or rescreen the data if new calibration information is available or special screening is needed in their analysis. 
The pipeline processes the data by sequence and, at the end of each run, the files within a sequence are packaged and sent to the HEASARC (USA) and DARTS (Japan) archives.

The archive is organized into directories, one for each sequence. Under each sequence directory there are directories for each instrument and one for the auxiliary data. The CAMS data and the MXS data are within the HXI and SXS directories, respectively. The different science data levels are stored in subdirectories within each of the instrument directory. Each sequence contains all the science, HK and auxiliary files for all the instruments and subsystems.

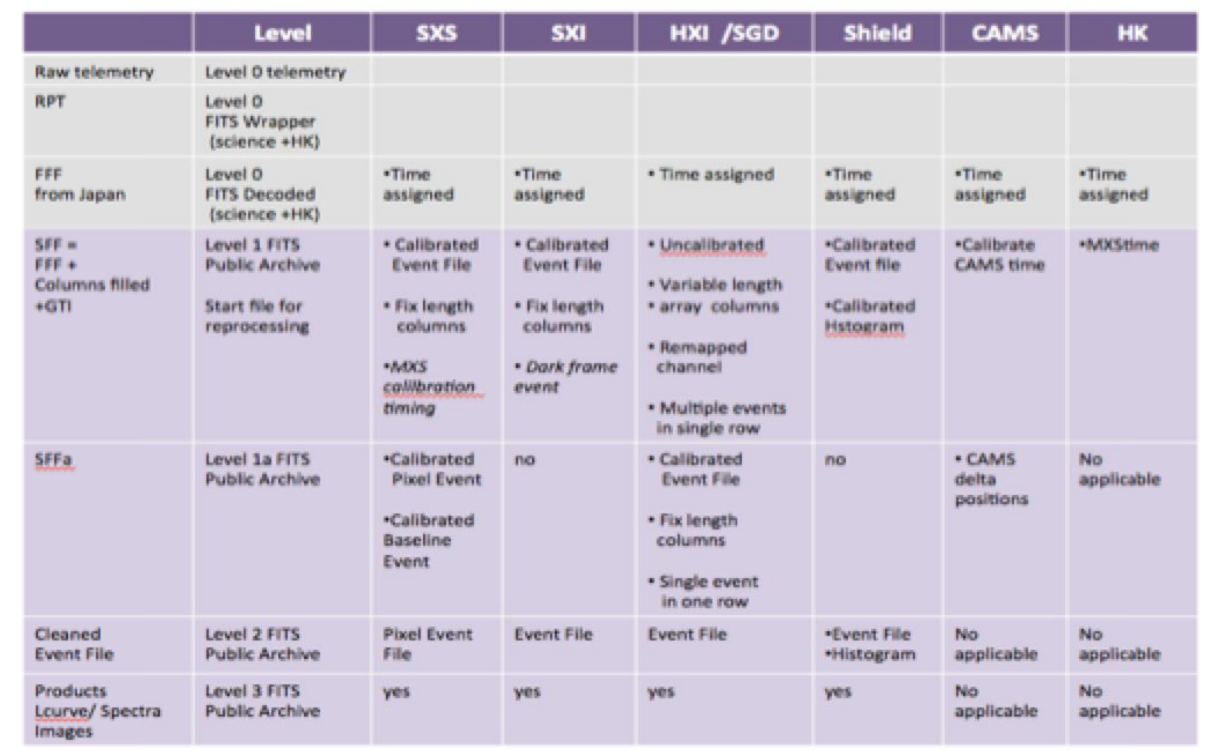

Figure 3. Data generated by the pre-pipeline are the RPT and FFF files (in the gray area). Date generated by the pipeline are the Level 1, 1a , 2, and 3 files (in the light purple area) and they populate the archive.

During the 38 days of operation, Hitomi observed six astronomical objects and made several blank sky observations. These data have been divided in about 40 sequences. These observations were taken during the commissioning phase and not all sequences have all the instruments operating.

\section{TIME, COORDINATES AND ENERGY ASSIGNMENT}

The following section describes the main software tasks written to calibrate the time, coordinates and energy for all instruments. These tasks are run in the data processing. The calibrated values are stored in columns of the FITS files. The column names using the analysis are TIME to record the time of the event, $\mathrm{X}$ and $\mathrm{Y}$ to record the pixel position in the sky frame and PI to record the channel number that is used to extract a spectrum. The FITS files for each instrument contains also other supporting columns that record previous steps necessary to derived TIME, $\mathrm{X}$ and $\mathrm{Y}$ and PI.

\subsection{Time assignment}

The time assignment framework is general for all science instruments and HK. Hitomi has a GPS receiver on board that dispatches the time to all subsystems as a TI (time indicator in unit of $2^{-6} \mathrm{sec}$ ). The instruments electronics assign the fine time to the science data. If the GPS is off, the time is assigned similarly as it was done for the Suzaku satellite where the TI is calibrated using the time assigned to the telemetry packets, the receiving time on ground and the temperaturefrequency relation of the on-board clock. 'ahmktim' and 'ahtime' are the main tasks in the Hitomi package used to assign time. Additional instrument-dependent tasks are used to pre-calculate the fine time. 'ahmktim' computes a table (TIM table) of TIME vs TI (the lower 32 bit of the TI value, L32TI) used by 'ahtime'. The calculation uses the combination of the GPS flags that indicate when the GPS is on, off, in transition or in illegal state. If the GPS is on, the TIME vs TI is obtained using the values of the time to when the packets are tagged (S_TIME) and the L32TI. If the GPS is off, TIME vs TI is calculated using the closest GPS time to derive the temperature of the on-board clock in the HK and the frequency-temperature relation obtained from the clock ground calibration. 'ahtime' computes the time for all science 
data and HK with a three steps procedure. The $1^{\text {st }}$ step derives the fine time resolution that is assigned internally by the instruments either using support instrument dependent look-up tables or keywords in the header of the FITS file. The $2^{\text {nd }}$ step derives, for a given L32TI, the corresponding time using the TIM table. The $3^{\text {rd }}$ step adds a delay, when appropriate, between the on-board computer and the instruments. For HK data the steps 1 and 3 are skipped because there is not need of fine time resolution. The times are stored in the column TIME which contains seconds since an epoch set to 2014-0101 00:00:00 UTC. The epoch is written in the header of all FITS files as an MJD value in the keywords MJDREFI and MJDREFF. The time system is TT (Terrestrial Time) and the MJD value of the epoch in TT corresponds to MJD 56658.0007775925926 (TT).

\subsection{Coordinates assignment}

Hitomi has three instruments at the focal plane of the telescopes: the SXI, SXS (soft energy telescopes) and the HXI (high energy telescopes). The coordinates for these instruments are described by five different coordinate systems and their values are stored in the FITS columns named PIXEL/RAWn, ACTn, DETn, FOCn, where $\mathrm{n}$ is either X or Y, and the columns $\mathrm{X} / \mathrm{Y}$ that represent the SKY system (see below). Since the SGDs are not imaging detectors, SGD files do not have any of the above columns for coordinates, however the XYZ position in the detector is provided for any reconstructed event. The transformation of coordinates for all instruments are defined in calibration files one for each instrument (teldef). The SXI, SXS and HXI coordinates are calculated by the task 'coordevt'. The pixel and the FOV sizes in the different coordinate systems are listed in Table 1.

Table 1. List for each coordinate system and detector : the pixel size, the number of pixels and if the coordinate definition is lookup/down with respect to the detector

\begin{tabular}{|c|c|c|c|c|c|c|c|c|c|}
\hline \multicolumn{2}{|c|}{ Instrument } & \multicolumn{2}{|c|}{$\begin{array}{l}\text { RAW/PIXEL } \\
\text { coords (look-down) }\end{array}$} & \multicolumn{2}{|c|}{$\begin{array}{l}\text { ACT coords } \\
\text { (look-down) }\end{array}$} & \multicolumn{2}{|c|}{$\begin{array}{l}\text { DET coords (look- } \\
\text { up) }\end{array}$} & \multicolumn{2}{|c|}{$\begin{array}{l}\text { FOC coords (look- } \\
\text { up) }\end{array}$} \\
\hline & & size & $\begin{array}{l}\text { pixel } \\
\text { arc sec }\end{array}$ & size & \begin{tabular}{|l} 
pixel \\
arc sec
\end{tabular} & size & $\begin{array}{l}\text { pixel } \\
\text { arc sec }\end{array}$ & size & $\begin{array}{l}\text { pixel } \\
\text { arc sec }\end{array}$ \\
\hline SXS & & $0: 35$ & -- & $1: 8,1: 8$ & 29.982 & $1: 8,1: 8$ & 29.982 & $\begin{array}{l}1: 2430 \\
1: 2430 \\
\end{array}$ & 1.768 \\
\hline \multirow[t]{3}{*}{ SXI } & segment & $\begin{array}{l}0: 639 \\
0: 319 \\
\end{array}$ & 1.768 & -- & -- & -- & -- & -- & -- \\
\hline & chip & -- & -- & $\begin{array}{l}1: 640, \\
1: 640\end{array}$ & 1.768 & -- & -- & -- & -- \\
\hline & array & -- & -- & -- & -- & $\begin{array}{l}1: 1810, \\
1: 1810\end{array}$ & 1.768 & $\begin{array}{l}1: 2430 \\
1: 2430 \\
\end{array}$ & 1.768 \\
\hline HXI-1 & & $\begin{array}{l}1: 128 \\
1: 128 \\
\end{array}$ & 4.297 & $\begin{array}{l}1: 256, \\
1: 256 \\
\end{array}$ & 4.297 & $\begin{array}{l}1: 256, \\
1: 256\end{array}$ & 4.297 & $\begin{array}{l}1: 2430 \\
1: 2430 \\
\end{array}$ & 1.768 \\
\hline HXI-2 & & $\begin{array}{l}1: 128, \\
1: 128\end{array}$ & 4.297 & $\begin{array}{l}1: 256 \\
1: 256\end{array}$ & 4.297 & $\begin{array}{l}1: 256, \\
1: 256\end{array}$ & 4.297 & $\begin{array}{l}1: 2430 \\
1: 2430\end{array}$ & 1.768 \\
\hline
\end{tabular}

The RAW coordinates comprise the basic coordinate system. For the SXS, they correspond to the telemetered pixel number where the event landed. This value is stored in the 1-d column PIXEL and ranges from 0-35. For the SXI, the RAW coordinates correspond to the telemetered event location into a segment of the CCD. Each CCD has two segments. The RAWY ranges from 0 to 639 and RAWX from 0 to 319. For the HXI, the RAW coordinates are calculated after the event is reconstructed using the telemetered strip location.

The ACT coordinates are derived from the RAW coordinates. The SXS ACT coordinates represent a 2-d look-down linearized coordinate system starting from the values stored in the PIXEL column. The SXI ACT coordinates correspond to the pixel locations in one SXI CCD. They range from 1 to 640 in the X and Y dimensions. The SXI RAW-to-ACT conversion depends on the window mode and readout node. The HXI ACT coordinates are the RAW coordinate corrected using the CAMS time-dependent misalignments to remove the Extended Optical Bench (EOB) movements, where the HXIs are located, respect to the main satellite body.

The DET coordinates are derived from the ACT coordinates. The SXI DET coordinates combine all four CCDs into a single system, accounting for any misalignments among them. The ACT-to-DET conversion changes the configuration from the look-down to the look-up for all instruments.

The FOC system combines all of the HXI, SXI, and SXS individual DET coordinates into a common system, accounting for misalignments among them. Since the SXI has the largest field of view and smallest detector pixel size, the FOC coordinates for all sensors adopt the pixel scale and range for the SXI.

The $\mathrm{X}$ and $\mathrm{Y}$ coordinates (or SKY) are derived from the FOC using the satellite attitude and they differ from the FOC for the position angle or roll. The $\mathrm{X}$ and $\mathrm{Y}$ columns contain pixel number. The pixel number is associated to a position in the sky via the FITS WCS keywords. The SKY system recorded the WCS keywords is a tangent plane projection and oriented such that declination $(\delta)$ increases in the $+Y$ direction and Right Ascension $(\alpha)$ increases in the $-\mathrm{X}$ direction. Figure 4 shows the in-flight sky images for the G21.5-0.9 source for each individual detector as well as the overlay of the HXIs and SXS onto the SXI frame. 


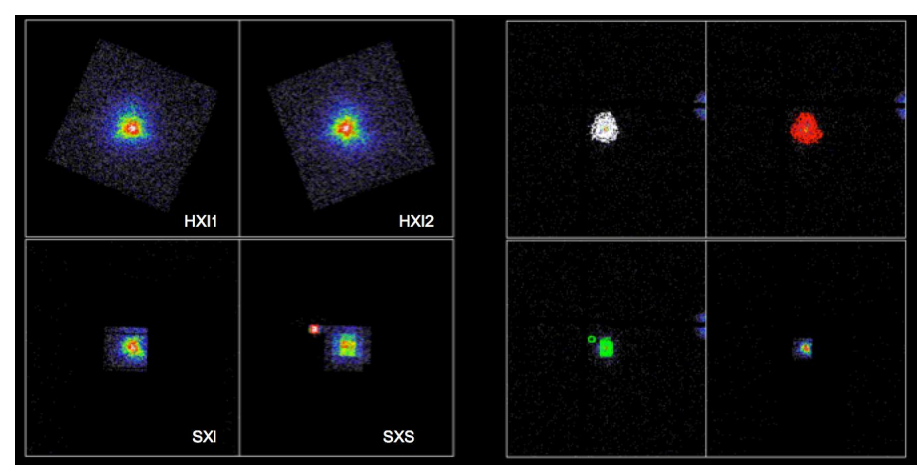

Figure 4. Left : Each panel shows the image of in an individual detector in sky coordinates. The bright red spot in the SXS image is the calibration pixel. Right: SXI Image with contour overlay of the HXI1, HXI2 and SXS respectively. SXI has the area discriminator on.

\subsection{Energy assignment}

The HXI, SGD, SXI, and SXS spectra are derived using the FITS column PI (pulse invariant) that corresponds to the linearized pulse height (channel) where each channel has equal energy width (see Table 2). The PI is calculated with algorithms appropriate for each instrument due to the different physics of the X-ray interaction with the detector. Many intermediate quantities are necessary before computing the PI value, and all tasks for the SXI, SXS , HXI and SGD records the intermediate values in additional columns of the event file.

Table 2. For each of the instrument PI_CHAN gives the PI channel range and PI_BIN is the energy for each PI channel in eV.

\begin{tabular}{|l|l|l|}
\hline Instrument & PI_CHAN & PI_BIN \\
\hline SXI & $0-4095$ & $6 \mathrm{eV}$ \\
\hline SXS & $0-32767$ & $0.5 \mathrm{eV}$ \\
\hline HXI & $0-2047$ & $0.1 \mathrm{keV}$ \\
\hline SGD & $0-2047$ & $0.75 \mathrm{keV}$ \\
\hline
\end{tabular}

The SXS telemetered 'energy' information is in the FITS column PHA for each primary and secondary event of any grade. The PHA of the secondary medium grade are first corrected by the task 'sxssecor' and written in the column PHA2 together with the telemetered PHA for primary events. The PI is calculated using 'sxsgain' and 'sxspha2pi' tasks. 'sxsgain' calculates the SXS time-dependent energy correction using a calibration line and derives the appropriate temperature for that correction. Using the results of 'sxsgain' and the SXS gain file, 'sxspha2pi' calculates the values for the PI column. The SXI telemetered 'energy' is stored in the 'PHAS' column that contains the $3 \times 3$ pixel array telemetered charge around the event. 'sxipi' calculates the PI column by correcting the values in the $3 \times 3$ array for the video temperature part of the CCD electronics, for the charge trail, and for the charge transfer inefficiency. Using the corrected $3 \times 3$ array, 'sxipi' assigns the grade and the pulse height for each event and populates the GRADE and PHA columns. In the last step, the gain is applied to each event and the computed pulse invariant stored in the PI column.

For the HXI and SGD the telemetered pulse height corresponds to the signals detected by the instrument. Since an X-ray photon when interacting with the detector may create more than one signal for one interaction there are many signals that may contribute to the final PI calculation. At the first step ' $h x i s g d p h a$ ' gain corrects the PHA of each individual signal, deriving an energy. The PI is calculated using 'hxievtid' and 'sgdevtid' tasks for the HXI and SGD, respectively. These tasks determines whether the signals due to the interaction of the X-ray with the detector parts are consistent with a valid event by comparing the detected signals with the allowed template patterns of signals. If an occurrence is consistent with an event, 'hxievtid' and 'sgdevtid' assign the PI to the reconstructed event considering the energies of the signals within the occurrence. 


\section{TELESCOPE EFFECTIVE AREA AND RESPONSE}

Figure 5 shows the SXS, SXI and HXIs Hitomi spectra extracted from a region centered on the G21.5-0.9 together with the best fit model. In order to model the data the spectral analysis program Xspec requires an ARF and a RMF files or a RSP file for each instrument. The RMF, ARF and/or RSP are calculated by software. The RMF is a spectral redistribution matrix (also known as the "line-spread function," or LSF), while the ARF is a multiplicative function of energy and contains all the information about the effective area (EA) due to the X-ray telescope, Quantum Efficiency (QE), and a number of other detector-related efficiencies. For the SXS and SXI there are tools to calculate both the ARF and RMP but because of the different detector characteristics for the HXI is only possible to calculate the net response matrix file (RSP). For the SGD the on-axis RSP are provided in CALDB and the software corrects for the off-axis transmission.

\subsection{SXI and SXS ARF and HXI RSP generator}

There is a common frame work to calculate the telescope effective area. 'aharfgen' is a script that calls different tasks to calculate the ARF for the SXS and SXI, and RSP for the HXI. Although the scheme for creating response functions differs among instruments, there are commonalities applicable to the three instruments. a) The landing position in the focal plane by event coming from a fix position in sky varies with the attitude movements. This variation is accounted by the outputs of the exposure calculation. 'ahexpmap' calculates an histogram of time spent at different off-axis positions and, for the SXI and SXS, also calculates the fractional exposure of each detector pixel for each off-axis bin. b) The telescope effective area is calculated by the ray-tracing, code which is essentially a Monte Carlo simulation of photons injected into the telescope aperture. The run is made for each off-axis angle and a pre-calculated energy grid. The output is an event file containing the path of each photon through the telescope and the final impact coordinates on the focal plane. c) The ray-tracing energy grid is coarser than the final energy grid of the ARF, because it utilizes optimized precalculated grid with fine energy sampling in regions where telescope or detector edges dominate and coarse sampling where no features are expected due to the telescope or the detector. d) There are four options to account for the spatial distribution of the X-ray source: point source, extended source flat within a circle, extended source described by a beta model or a spatial distribution from an image obtained by different mission. Events generated by the ray-tracing are filtered through the source region selection and propagated down to the detector. Any object encountered by the event down to the detector is accounted at this stage. These are the filters for SXS and SXI and baffle for the HXI.

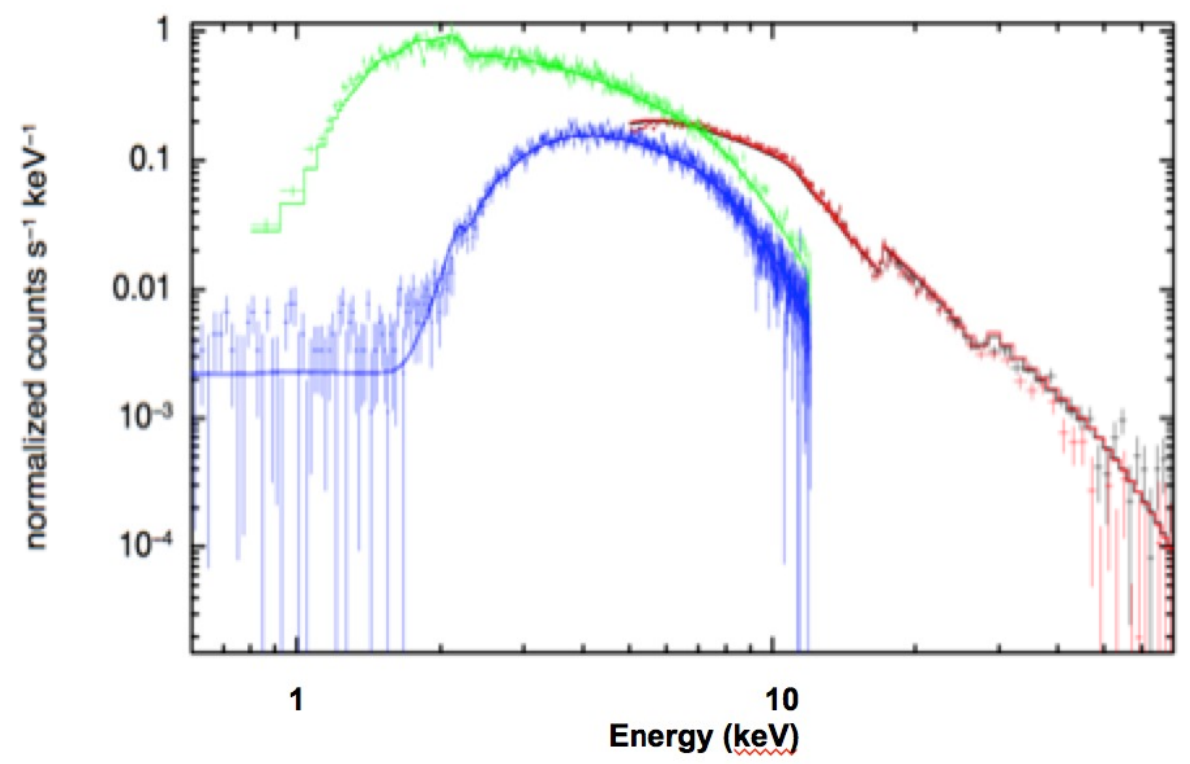

Figure 5. G21.5-09 spectra of the SXI (green), SXS (blue), HXI1 (black) and HXI2 (red) with the best fit model overlaid. 
For the SXI and SXS the final ARF calculation also includes: the quantum efficiency that is position dependent for the SXI but not for the SXS, the contamination and the exposure information. For the HXI the code outputs an RSP because of the intrinsic detector characteristics that are also time-position dependent. The LSF and QE are different for each of the five layers present in a single HXI unit. The HXI is located in the EOB that moves with respect to the main satellite body where the telescopes are located. The EOB movements are tracked by the CAMS data. Therefore the net response matrix is a weighted combination of the five responses. The weights depends on the relative time-position dependent $\mathrm{X}$ ray source counts, which in turn depends on the movements of the EOB with respect to the telescope and on the telescope effective area.

\subsection{SXI and SXS RMF generator}

The RMF for the SXS and SXI are calculated by the 'sxsmkrmf' and 'sxirmf' tasks, respectively. The SXS RMF depends on the grade distribution and the pixels selected to create the spectrum. 'sxsmkrmf' first calculates an histograms of grades for the pixels of interest and then calculates the RMF for each pixel and grade and averages the individual RMF according to the histogram. By default, the SXS RMF input and output grids result in an RMF with 32768 channels of width $0.5 \mathrm{eV}$. The RMF for the SXI depends on the extraction region and 'sxirmf' uses the WMAP, a coarsebinned image of the extraction region with the spatial counts distribution, to determine the weight distribution for the LSF from different positions on the detector. By default, an RMF is generated with an input energy grid in the range 0.200 to $23.974 \mathrm{keV}$, and with 5900 output channels of width $2 \mathrm{eV}$ up to $\sim 12 \mathrm{keV}$ and 500 channels up to $\sim 24 \mathrm{keV}$.

\section{SUMMARY}

The Hitomi software package and calibration data are in distribution with the HEAsoft package and CALDB both available from HEASARC. The Hitomi data populate the public archive at the HEASARC and DARTS after one year propriety period that starts when the final data processing is completed. Updates on software, calibration and data availability are posted at http://heasarc.gsfc.nasa.gov/docs/hitomi/.

\section{REFERENCES}

[1] Takahashi, T., Mitsuda K., Kelley, R.L., et al., “The Astro-H X-ray astronomy satellite”, Proc SPIE 9144, 994125 (2014)

[2] Mitsuda, K., et al. "Soft x-ray spectrometer (SXS): the high-resolution cryogenic spectrometer onboard ASTROH", Proc. SPIE 9144, 773211-773211-10 (2014)

[3] Soong, Y., et al. "ASTRO-H Soft X-ray telescope (SXT)", Proc. SPIE 9144, 994128-914425-14 (2014)

[4] Hayashida, K., et al., "Soft x-ray imager (SXI) onboard ASTRO-H", Proc. SPIE 9144, (2014)

[5] Sato, G., et al., "The hard x-ray imager (HXI) for the ASTRO-H mission", Proc. SPIE 9144 , (2014)

[6] Awaki , H., et al., "ASTRO-H Hard X-ray telescope (HXT) ', Proc. SPIE 9144 , (2014)

[7] Fukazawa, Y., et al., "Soft gamma-ray detector for the ASTRO-H Mission", Proc. SPIE 9144 , (2014)

[8] Gallo, L., et al., 'The Canadian ASTRO-H Metrology System", Proc. SPIE 9144, (2014)

[9] de Vries ,C. P., et al., "Calibration sources for the soft x-ray spectrometer instrument on ASTRO-H", Proc. SPIE 8443, (2012) 